\title{
Roughness Based Crossflow Transition Control: A Computational Assessment
}

\author{
Fei Li", Meelan Choudhari ${ }^{*}$, Chau-Lyan Chang ${ }^{*}$, Craig Streett ${ }^{*}$, and Mark Carpenter \\ NASA Langley Research Center, Hampton, VA 23681
}

\begin{abstract}
A combination of parabolized stability equations and secondary instability theory has been applied to a low-speed swept airfoil model with a chord Reynolds number of 7.15 million, with the goals of (i) evaluating this methodology in the context of transition prediction for a known configuration for which roughness based crossflow transition control has been demonstrated under flight conditions and (ii) of analyzing the mechanism of transition delay via the introduction of discrete roughness elements (DRE). Roughness based transition control involves controlled seeding of suitable, subdominant crossflow modes, so as to weaken the growth of naturally occurring, linearly more unstable crossflow modes. Therefore, a synthesis of receptivity, linear and nonlinear growth of stationary crossflow disturbances, and the ensuing development of high frequency secondary instabilities is desirable to understand the experimentally observed transition behavior. With further validation, such higher fidelity prediction methodology could be utilized to assess the potential for crossflow transition control at even higher Reynolds numbers, where experimental data is currently unavailable.
\end{abstract}

\section{Introduction}

Substantial reductions in the fuel burn of future aircraft have been targeted both in the U.S. [1] and in Europe $S_{\text {[2] }}$, in order to lower the cost of air travel and alleviate the impact of aviation on the environment. A substantial fraction of the targeted reductions in aircraft fuel burn will accrue from the reductions in vehicle drag. For today's commercial transport aircraft, up to one half of the total drag corresponds to skin friction drag. Because laminar skin friction is much lower in comparison with the turbulent skin friction, flow control via delayed boundary-layer transition over major aerodynamic surfaces holds the potential to provide the desired reductions in the overall drag.

NASA's Subsonic Fixed Wing Project is investigating several options to help develop robust technology concepts for laminar flow control and to accelerate their insertion into operational fleet [1]. One of those concepts involves the delay of crossflow-induced transition over a swept aerodynamic surface using control input in the form of appropriately positioned Discrete Roughness Elements (DREs) near the leading edge [3]. A bulk of the existing experimental and computational studies of the DRE concept have been carried out for either incompressible or supersonic configurations with modest wing-chord Reynolds numbers of up to approximately 8 million $[4,5]$, and with pressure distributions that may or may not be optimal for wing designs for subsonic transport aircraft flying at Mach numbers between 0.75 to 0.90 . Consequently, additional effort will be necessary to assess the potential capability of the DRE concept to control swept wing transition at transonic Mach numbers and substantially higher chord Reynolds numbers than previous applications.

Given the sensitivity of transition to wind tunnel disturbances and the cost of flight experiments, computational tools will need to play an important role in the assessment of DRE for subsonic transport vehicles, as a means of risk reduction as well as to help optimize the DRE design. The DRE concept seeks to delay transition via controlled seeding of subdominant crossflow modes that cannot lead to transition on their own, but can keep the naturally dominant instability modes at bay via nonlinear modification of the basic state and/or mode competition. This intrinsically nonlinear control mechanism calls for a higher fidelity prediction approach involving most, if not all, of the relevant stages of the transition process, namely, (1) excitation of primary crossflow instability, (2) linear and nonlinear development of the primary crossflow instability, and (3) excitation, linear growth and nonlinear breakdown of high-frequency secondary instability [6]. A number of individual ingredients of this approach have

Aerospace Technologist, Computational AeroSciences Branch 
been available for some time and partial integration of those ingredients has been demonstrated in prior works [6-10]. The important missing parts are the receptivity and the nonlinear breakdown of secondary instability, the latter of which directly leads to the onset of transition as manifested by a sudden rise in the wall shear-stress.

The objective of the present work is to utilize a combination of parabolized stability equations and secondary instability theory, to (1) assess the effectiveness of DRE control and (2) compute the nonlinear development and breakdown of secondary instability up to the onset of transition. The capability to carry out the latter task implies that the high fidelity, fully integrated transition prediction tool is one step closer to reality.

The receptivity of the stationary crossflow vortex and that of the secondary instability will not be discussed in this paper. A number of investigations in existing literature have addressed the former problem (e.g. [9]), but the latter problem does not appear to have been tackled so far. Thus, parametric studies encompassing a range of initial amplitudes of the stationary crossflow vortex as well as the secondary instability modes are used to gauge the resulting variation sin transition characetristics. The rest of the paper is laid out as follows. Section II briefly describes the flow configuration under examination and the computational codes used during the present effort. In Section III, the results of stationary crossflow computations are presented and the effect of control mode on target mode evolution is analyzed. In section IV, results of linear secondary instability computations are presented and the effects of control on linear growth characteristics of the secondary modes are discussed. In section V, PSE computations of the nonlinear development of secondary instability modes, the ensuing breakdown process and transition criteria accounting for the secondary instability characteristics are discussed. Finally, a summary of the paper is given in Section VI.

\section{Flow Configuration and Analysis Codes}

The flow configuration examined herein corresponds to the SWIFT flight test article from [4]. A 30-degree swept airfoil was mounted vertically beneath the wing of a Cessna O-2A Skymaster aircraft and flown at chord Reynolds numbers of 7 to 8 million, in order to investigate the feasibility of DRE technology for SensorCraft applications. The mean flow around the installed configuration was computed via CFD [5]. Spanwise variations in the computed pressure distribution over the mid-span segment of the suction surface were relatively small, so that the region in between the rows of static pressure ports at 13 inch and 29 inch span locations outboard of the mounting plane approximated (locally) infinite span conditions. Transition measurements, both without and with the use of DRE, were reported in [4].

All of the results presented in this paper correspond to the inboard measurement location at Test Point 27 [3]. For this case, the Reynolds number $\mathrm{Re}_{\mathrm{c}}$ based on the free-stream velocity and the streamwise chord length is equal to $7.15 \times 10^{6}$ and the angle of attack over the test article is -4.69 degrees. The mean flow at these conditions computed with a boundary layer solver [12] using infinite span approximation, in conjunction with the sectional surface pressure distribution derived from the CFD solution [5]. Linear and nonlinear development of the instability modes is computed using parabolized stability equations (PSE) as implemented in the Langley Stability and Transition Analysis Codes (LASTRAC) [13]. Linear secondary instability predictions are made using the methodology outlined in [10]. Nonlinear development and breakdown of secondary instability are again computed using LASTRAC. The selection of grid parameters and other aspects of the numerical solution was based on extensive experience with such calculations for similar classes of flows $[6,7,9,10]$, and spot checks were made to ensure that any variations with respect to modifications to those choices were sufficiently small.

\section{Stationary Crossflow Vortices and Effect of Control}

Linear amplification characteristics of stationary crossflow instability at the flow conditions of interest have been described in [5]. Specifically, the crossflow mode with a spanwise wavelength of $\lambda=4.5 \mathrm{~mm}$ corresponds to the maximum logarithmic amplification ratio, $\mathrm{N} \approx 12$, based on linear stability theory. The first harmonic of the above mode $(\lambda=2.25 \mathrm{~mm})$ has a considerably lower linear growth potential $(\mathrm{N} \approx 5.6)$ and, therefore, was used to control the growth of the more amplified instability modes via DRE.

Flight observations under somewhat different conditions $\left(\mathrm{Re}_{\mathrm{c}}=8.1 \times 10^{6}\right.$ and $\left.\mathrm{AOA}=-4 \mathrm{deg}\right)[4]$ had shown that, with a painted leading edge (average roughness height of $1.0 \mu \mathrm{m} \mathrm{rms}$ and $3.8 \mu \mathrm{m}$ average peak-to-peak), the natural onset of transition at the spanwise section of interest was near $x / c \approx 0.3$, whereas transition was delayed beyond $x / c$ $\approx 0.8$ when the leading edge surface was unpainted and, therefore, was considerably smoother (r.m.s. roughness height of $0.3 \mu \mathrm{m} \mathrm{rms}$ and $2.2 \mu \mathrm{m}$ average peak-to-peak). Even with the painted leading edge, transition onset was 
pushed back to $x / c \approx 0.6$ when a $12 \mu \mathrm{m}$ height roughness array with $2.25 \mathrm{~mm}$ spacing was introduced just downstream of the airfoil leading edge.

It is reasonable to assume that both the painted and polished leading edges of the test article had a broadband surface roughness distribution, with a wavenumber spectrum that overlapped with the previously discussed range of most unstable stationary crossflow modes. A match in length scales would lead to a direct excitation of a correspondingly broad spectrum of unstable modes via a linear receptivity mechanism [14]. Of course, following sufficiently strong (and differential) amplification over a finite downstream distance, the disturbance spectrum would eventually tend be dominated by the modes in the vicinity of the linearly most amplified wavelength. As a simple, conceptual model for the effects of background surface roughness on transition via the dominant range of wavelengths, we first consider the streamwise evolution of a stationary crossflow mode with $\lambda=5 \mathrm{~mm}$ and a range of initial amplitudes (Fig. 1a). Analogous evolution of crossflow modes with shorter and longer wavelengths, respectively, is shown in Figs. 1b and 1c, respectively. The limited range of chordwise locations included in each plot is due to the beginning of a strongly adverse gradient slightly downstream of $x / c=0.70$, which leads to flow separation within the framework of boundary layer equations. It is interesting to observe that, regardless of the disturbance wavelength, crossflow disturbances with sufficiently large initial amplitude exhibit a peak in the fundamental amplitude, followed by a significant decrease in fundamental amplitude in the immediately downstream region. In other words, no extended regions of quasi-saturation observed for the lower Reynolds number, NLF-0415(b) configuration $[3,7]$ are noted in the present case.

Figure 2 illustrates a typical sequence of velocity contours during the nonlinear evolution of an unstable crossflow mode. The initially undulating contours give way to strongly overturned contours that are symptomatic of strongly inflexional velocity profiles in the outer part of the boundary layer and, hence, the onset of high-frequency secondary instabilities that typically, but not always, harbinger the onset of laminar breakdown to turbulence.

We now consider the scenario when the $\lambda=2.25 \mathrm{~mm}$ mode with a larger initial amplitude is introduced as the control input for its more unstable subharmonic with $\lambda=4.5 \mathrm{~mm}$. We note here that the term "amplitude" is defined as the maximum perturbation velocity in the normal chord direction (i.e. parallel to the leading edge) as a fraction of the freestream velocity. The combined evolution of the control and target modes with the initial amplitude of the 4.5 mm mode held fixed at $10^{-4}$ (based on the preceding discussion for the $5 \mathrm{~mm}$ mode) is shown in Fig. 3(a). As the amplitude of the control input is increased from 0 to 0.01 , the amplitude levels of the target mode within the downstream region become substantially smaller. Of course, the peak amplitudes of the control mode also increase with the amplitude of the initial control input, which introduces the possibility of the control mode sustaining highfrequency secondary instabilities and, hence, precipitating an earlier onset of transition. This possibility will be discussed in the subsequent sections of this paper.

The amplitude curves plotted on a logarithmic scale (Fig. 3(b)) indicate that the control input does not begin to influence the growth of the target mode until the amplitude of the control mode has become sufficiently large as to modify the basic state. In the vicinity of the peak amplitude of the control mode (which is also where the mean flow distortion due to the control mode is the greatest), the growth rates of the target mode become relatively smaller, accounting for the stabilization of this mode. As shown in Fig. 4(a), at least part of the stabilization may perhaps be attributed to the reduced crossflow Reynolds numbers in the presence of the control mode with nonlinear amplitudes [15]. However, the same control input appears to result in larger values of $R_{c f}$ farther downstream; therefore, the mechanism underlying the stabilization of the target mode cannot be attributed to changes in $R_{c f}$ alone. Figure $4(b)$ compares the linear $\mathrm{N}$-factors of the target mode as predicted via instability analysis of the spanwise averaged mean flow without and with the control input. The figure shows that the modified basic state leads to an $\mathrm{N}$-factor for the target mode that is smaller in comparison with the unperturbed flow by approximately 3.2.

\section{Linear Secondary Instability and Effect of Control}

For stationary crossflow vortex instability, the dominant velocity component is that along the vortex axis and the velocity components in the cross-plane normal to the vortex axis are relatively small in comparison. In some of the previous investigations (e.g. [16]), the flow within the cross-plane has, nonetheless, been found to have a large influence on the growth rates of high frequency secondary instability supported by the finite amplitude primary crossflow modes. To assess the importance of the cross-plane flow, the secondary instability characteristics were computed both with and without the cross-plane velocity components associated with the distorted mean flow. The 
secondary instability growth rates at $x / c \approx 0.45$ are shown in Fig. 5 (a) for a primary crossflow vortex with $\lambda=5.0$ $\mathrm{mm}$ and an initial amplitude of $10^{-4}$. Clearly, those components appear to have a rather small effect in this particular case. It is found that there are multiple families of unstable secondary modes, but only the three most dominant ones are shown in Fig. 5(a). Representative mode shapes for these dominant modes are shown in Fig. 5(b). The included labels for each family $(Y, Z$, and $Y / Z)$ indicate whether the wall-normal and/or the spanwise shear associated with the distorted basic state (Fig. 2) dominates the energy production mechanism associated with a given family of secondary instability modes [7]. The $Y / Z$-mode looks like a $Y$-mode in the sense that it rides on top of the stationary crossflow vortex with a mode shape similar to that of a $Y$-mode (see bottom of Fig. 2(b)); however, it is categorized as a mixed $Y / Z$-mode because its energy balance reveals that the contribution to its growth rate from the production term associated with the spanwise shear is comparable to the contribution due to wall-normal shear.

The spatial amplification characteristics of various fixed frequency secondary instabilities belonging to each of the three dominant families of secondary instability modes are shown in Fig. 6. It is observed that the secondary modes grow extremely rapidly in comparison with the finite amplitude primary crossflow modes, achieving (secondary) $\mathrm{N}$ factors in excess of 9 across a short distance of around 6 percent chord. Of course, the receptivity characteristics of each of these modes will determine whether or not that mode gets excited with an adequately large initial amplitude in order to have a visible influence on the transition process. For example, previous experimental studies $[17,18]$ indicate a preferential excitation of the $z$ modes under certain conditions. Given this scenario, it seems prudent to track the $\mathrm{N}$-factor envelopes for each mode family separately rather than a single envelope for all secondary instability modes.

The effect of control on primary stationary crossflow vortices has been discussed in the previous section. The associated evolution of secondary instability is studied here. With the initial amplitude of the $4.5 \mathrm{~mm}$ primary stationary crossflow vortex mode fixed at $10^{-4}$, the effect of increasing the amplitude of the $2.25 \mathrm{~mm}$ control mode is shown in Figure 7. With zero control amplitude, it is seen that both the dominant $Y$-mode and $Z$-mode undergo strong modal amplifications and that the difference in estimated transition onset location would be $\Delta x / c=\mathrm{O}(0.05)$ depending on whether the $Y$ or the $Z$ modes are chosen for transition correlation.

As the control amplitude increases, modal amplifications of both the dominant $Y$ - and $Z$-modes are reduced. As shown in Figure 7, the presence of the control mode apparently affects the $Z$-mode of secondary instability much more than the $Y$-mode. This indicates that, if indeed transition is caused by the growth of the $Z$-mode as some experiments seem to suggest $[17,18]$, the DRE is a very effective means to achieve transition delay in the present case.

Note that the mixed $Y / Z$ mode has been conspicuously missing from the above discussion of the effect of control input. Without control, the $Y / Z$ mode is subdominant to the $Y$-mode. With increasing control amplitudes, this mode is also suppressed. An examination of its energy balance shows that the contribution from the part of the production term associated with the spanwise shear becomes negligible or even negative, i.e., the mixed mode now transforms into a predominantly $Y$-mode and is always subdominant to the most unstable $Y$-mode shown in Figure 7 . For all practical purposes, no special attention needs to be paid to this mixed $Y / Z$ mode, except to note that the morphing from a mixed $Y / Z$-mode to a pure $Y$-mode is further evidence that, at the present set of flow conditions, the DRE is more effective in suppressing the instability mechanisms associated with the spanwise shear.

While introducing the subdominant crossflow mode at relatively larger initial amplitudes may lead to substantial reduction in the growth of the otherwise dominant crossflow mode and, hence, in the growth potential of the secondary instability modes associated with the latter mode, the possibility of overcontrol (i.e., risk of premature transition due to the finite amplitude control mode) cannot be eliminated. That is, in this case, the $2.25 \mathrm{~mm}$ control mode could become susceptible to secondary instability and may itself break down to cause an earlier onset of transition. Secondary instability analysis for the $2.25 \mathrm{~mm}$ control mode is carried out to assess the likelihood of such a scenario. The peak $\mathrm{N}$-factors for Z-mode of secondary instability are less than 1 for all three control inputs. Of the three initial amplitudes of 0.002, 0.005 and 0.01 , only the last gives rise to a maximum $Y$-mode secondary instability $\mathrm{N}$-factor of approximately 11 at a chordwise location close to $x / c=0.25$. For the other two amplitudes of control input, the $Y$-mode $\mathrm{N}$-factors remain less than or equal to approximately 8 . Therefore, even if the $Y$-mode can also lead to transition, the possibility of premature transition due to over control is remote as long as the control amplitudes are kept under a certain threshold, which in this case appears to be around 0.005 or slightly larger. 


\section{Nonlinear Development and Breakdown of Secondary Instability}

As described in section IV, the linear predictions of secondary instability may be used for transition onset correlation using a secondary $\mathrm{N}$-factor criterion. However, it is also instructive to study the nonlinear evolution of the secondary mode perturbations in order to study the breakdown mechanisms responsible for the onset of transition. Wassermann and Kloker [19] carried out direct numerical simulations (DNS) of secondary instability well into the breakdown region and revealed some interesting features of the breakdown process for a specific wind tunnel configuration. Although DNS is a powerful tool, it is also costly. The present section investigates the application of nonlinear parabolized stability equations (PSE) to capture the initial portion of the breakdown process at a computational cost that is at least an order of magnitude lower in comparison with the DNS. Illustrative PSE computations of the breakdown of crossflow vortices with $\lambda=5 \mathrm{~mm}$ via the secondary instability mechanism are presented below.

The secondary instability eigenfunction is computed at a short distance downstream of the neutral point predicted by the linear, 2-D eigenvalue analysis, and is fed into the nonlinear PSE computation as initial conditions at that location. Figure 8 shows the comparison between the secondary instability growth obtained via PSE and linear theory, respectively. The left half of Figure 8 shows the evolution of a Y-mode of secondary instability at the frequency of $f=36 \mathrm{kHz}$, whereas the right half shows similar comparison for a Z-mode at $f=32 \mathrm{kHz}$. The red and green lines represent, respectively, the amplitudes of stationary crossflow vortices and secondary instability at different spanwise wave numbers obtained by PSE computations. The black dots represent the linear growth predictions based on the secondary instability theory. It is seen that, when the secondary instability amplitude is small, PSE results and the quasi-parallel linear theory agree very well for the Z-mode. For the $Y$-mode, there is some initial discrepancy between the linear theory and PSE results. The reason for this is currently unclear, but the PSE solution is known for having initial transient behavior when the inflow condition deviates from the exact nonparallel eigensolution. In any case, after the transient dies out, the PSE solution again agrees very well with the secondary instability theory.

The transition location can be correlated to either the amplitude of the nonlinearly developing secondary instability or the rise in wall-shear stress that characterizes the onset of transition. As discussed before, experimental evidence suggests that the crossflow vortex breakdown tends to occur via the Z-mode of secondary instability [17, 18]. Thus, a parametric study of the transition onset location as a function of the initial amplitude of secondary instability is carried out. Figure 9 shows the normalized wall shear stress (red lines), together with the RMS amplitude (green lines) of the secondary instability for several initial amplitudes of the Z-mode. The left most shear and amplitude curves correspond to an initial chordwise velocity perturbation of 0.01 , and successively adjacent curves to the right are associated with lower initial amplitudes in 10-fold decrements. In all cases, as the secondary instability amplitudes become sufficiently nonlinear, the wall-shear curve starts to rise and becomes nearly vertical at a short distance downstream. This rapid rise in wall shear is symptomatic of the onset of transition. If the transition onset location is to be based on the location where the wall-shear stress rises sharply, then the uncertainty in the predicted transition onset location (indicated by the dashed vertical in Fig. 9) is very small provided that the initial amplitude of the secondary instability is known.

The transition onset location could also be defined in terms of the location where the root mean square (RMS) amplitude of the secondary instability exceeds a certain threshold value. However, this transition criterion may be somewhat less robust due to the fact that the RMS amplitude does not rise as sharply as the wall shear and, in some cases, also undergoes a drop-and-rise in the vicinity of the transition onset (see green lines in Figure 9).

It can be further noted in Figure 9 that, with each increase in initial amplitude of the most amplified secondary instability mode by a factor of 10, the transition location based on, say, the wall-shear criterion moves upstream by approximately equal intervals. The red line in Figure 10 represents the transition location as a function of the logarithm of the initial amplitude of the secondary instability is almost a straight line, at least in this particular case. This appears to indicate that the nonlinear effect due to the amplification of higher harmonics is limited to very short streamwise domain just upstream of the transition onset. The implication is that linear secondary instability $\mathrm{N}$-factor methods, together with known initial amplitude of the secondary perturbation, may be sufficient to predict the 
transition location with reasonable accuracy. Ref. [20] describes linear amplitude criteria based on primary instabilities and their application for transition prediction.

Suppose that the transition onset is correlated with a constant linear amplitude of $A_{t r}$. Then, by linear theory, the relation between the initial amplitude and the $\mathrm{N}$-factor at transition is,

$$
A_{t r}=A_{i} e^{N_{t r}}
$$

where $A_{i}$ and $N_{t r}$ represent the initial amplitude and the transition $\mathrm{N}$-factor, respectively. Because $A_{t r}$ is assumed to be constant, it follows that

$$
\frac{\Delta N_{t r}}{\Delta \log \left(A_{i}\right)}=-1,
$$

i.e., the slope of the $N_{t r}$ vs. $\log \left(A_{i}\right)$ curve is equal to -1 .

The linear $\mathrm{N}$-factors at the transition locations resulting from the $\mathrm{Z}$-mode at $33 \mathrm{kHz}$ are also plotted (represented by the green line) in Figure 10. The curve is approximately a straight line whose slope (represented by the dashed line) is very close to that given by $\mathrm{Eq}(2)$.

Figure 11 shows the RMS amplitude distributions associated with the high frequency instability at selected chordwise locations. At each station, the amplitude distribution is normalized so as to have a maximum value of unity. For small values of $x / c$, the amplitude distributions mimic the linear eigenfunctions shown in Figure 3 and remain so over much of the region between the locations where the secondary instability is initiated and where the transiton onset occurs. Very close to the onset of transition, the amplitude distributions begin to be less concentrated and start to approach the wall, eventually leading to the sharp rise in spanwise averaged wall shear.

In the results presented thus far, the visualization of secondary instability were limited to 1- or 2-dimensional representations. The descriptions below are intended to show a rudimentary 3-dimenional picture pertaining to the nonlinear growth of secondary instabilities. Without secondary instability, the U-iso-surfaces for purely stationary crossflow vortices would look like smooth tubes that are approximately aligned with the boundary-layer edge velocity. With the introduction of the secondary instability, these iso-surfaces are modified. The top and bottom portions of Fig. 12 depict the instantaneous iso-surfaces of chordwise velocity, $U$, for the cases initiated with $Y$ - and $Z$-modes of secondary instability, respectively. The initial amplitudes of the secondary instability modes for both cases in Fig.12 are equal to 0.01. The "Rib-like" structures previously observed in [19] and [21] are clearly visible in both cases. By examining the vorticity of these "ribs", it can be determined that these are, in fact, co-rotating vortices which ride on the top of the stationary crossflow vortex in the case of $Y$-mode, or on the side of the stationary vortex in the case of Z-mode of secondary instability. Furthermore, these "ribs" align themselves at an oblique angle relative to the axis of the stationary crossflow vortex (see, also, ref. [19] in this context). Farther downstream, smaller structures begin to appear, which should eventually lead to turbulence.

\section{Summary}

The present effort targets the development, application, and improvement of higher fidelity tools for DRE based laminar flow control over subsonic aircraft. The results obtained for the SWIFT flight configuration demonstrate that useful insights may be gained into the efficacy and/or optimization of a given DRE application using such tools.

Computations of the nonlinear evolution of stationary crossflow modes using the parabolized stability equations reveal that, with increasing amplitude of the control mode with $\lambda=2.25 \mathrm{~mm}$, a corresponding reduction in the amplitude of the target mode with $\lambda=4.5 \mathrm{~mm}$ is achieved.

The reduction in the target mode amplitude is accompanied by reduced linear growth rates of the secondary instabilities. Several secondary instability modes are found, of which three representative ones with the largest growth rates are analyzed. It is revealed that the presence of the control modes reduces the growth of the Z-mode secondary instability much more effectively than the $Y$-modes.

The possibility of over-control is also assessed, and it is found that, if the $Y$-mode can be a catalyst for transition, initial control amplitude larger than a certain threshold may make the control mode itself susceptible to 
secondary instability breakdown, leading to earlier-than-intended transition at the flow conditions investigated in the paper. However, if the Z-mode alone is responsible for transition, the threshold amplitude is significantly increased.

The nonlinear development of the most unstable Z-mode secondary instability is traced using the PSE method. The PSE results agree well with linear secondary instability when the amplitude is small. At large amplitudes, a sharp rise in the wall-shear stress indicates the onset of transition. If transition onset prediction is based on the location of the sharp rise in wall-shear stress, the uncertainty in transition onset location is very small.

The role of low frequency, traveling crossflow instabilities during the transition process has not been discussed in this paper. It is known that, in the presence of strong stationary crossflow vortices, traveling crossflow instabilities are modulated [7, 10], with maximum amplitude nearer to the wall than the secondary instabilities. Therefore, future studies may include the development of modulated low frequency, traveling crosssflow instabilities and their interaction with high frequency secondary instabilities.

\section{Acknowledgments}

The authors are thankful to Prof. William Saric of Texas A \& M University for providing the geometry and surface pressure distributions for the SWIFT flight configuration. The work described in this paper has been carried out under Subsonic Fixed Wing project of NASA's Fundamental Aeronautics Program.

\section{References}

1. Collier, F., "Subsonic Fixed Wing Project Overview," Proceedings of NASA FAP 2008 Annual Meeting, Atlanta, Oct. 7-9, 2008.

2. Report of the Group of Personalities, "European Aeronautics: A Vision for 2020," January 2001.

3. Saric, W. S., Carillo, R. B., and Reibert, M. S., "Leading Edge Roughness as a Transition Control Mechanism," AIAA Paper 98-0781, Jan. 1998.

4. Carpenter, A.L., Saric, W.S., and Reed, H.L., "Laminar Flow Control on a Swept Wing with Distributed Roughness," AIAA Paper 2008-7335, 2008.

5. Rhodes, R.G., Carpenter, A.L., Reed, H.L., and Saric, W., "CFD Analysis of Flight-Test Configuration for LFC on Swept Wings," AIAA Paper 2008-7336, 2008.

6. Choudhari, M., Chang, C.-L., Streett, C. L., and Balakumar, P., "Integrated Transition Prediction: A Case Study in Supersonic Laminar Flow Control," AIAA Paper 2003-0973, 2003.

7. Malik, M.R., Li, F., Choudhari, M., and Chang, C., "'Secondary Instability of Crossflow Vortices and Swept-Wing Boundary-Layer Transition," J. Fluid Mech., vol. 399, pp. 85-115, 1999.

8. Janke, E., "Receptivity and Transition Control of Swept-Wing Boundary-Layers; Effects of Surface Curvature and Nonlinearity," AIAA Paper 2001-2980, 2001.

9. Chang, C.-L. and Choudhari, M. M., "Boundary-Layer Receptivity and Integrated Transition Prediction," AIAA Paper 2005-0526, 2005.

10. Li, F., and Choudhari, M., "Spatially Developing Secondary Instabilities and Attachment Line Instability in Supersonic Boundary Layers," AIAA Paper 2008-590, 2008.

11. "Excitation of Crossflow Instabilities in a Swept Wing Boundary Layer," Submitted to 39 ${ }^{\text {th }}$ AIAA Fluid Dynamics Conference, San Antonio, June 2009.

12. Wie, Y.-S., "BLSTA - A Boundary Layer Code for Stability Analysis," NASA CR 4481, 1992.

13. Chang, C.-L., "Langley Stability and Transition Analysis Code (LASTRAC) Version 1.2 User Manual," NASA/TM-2004213233, June, 2004.

14. Choudhari, M., "Roughness-Induced Generation of Crossflow Vortices in Three-Dimensional Boundary Layers," Theor. and Comp. Fluid Dyn., Vol. 5, pp. 1-31, Feb. 1994.

15. Choudhari, M., Chang, C.-L., and Jiang, L., "Towards Transition Modeling for Supersonic Laminar Flow Control," Philosophical Transactions of Royal Society of London (Physical and Mathematical Sciences), vol. 363, no. 1830, pp. 10411259, 2005.

16. Bonfigli, G, and Kloker, M., "Secondary Instability of Crossflow vortices: validation of the stability theory by direct numerical simulation,” J. Fluid Mech., Vol. 583, pp. 229-272, 2007.

17. Kawakami, M., Kohama, Y. \& Okutsu, M. "Stability Characteristics of Stationary Crossflow vortices,", AIAA Paper 990811, 1999.

18. White E. B., and Saric W. S., "Secondary Instabilty of Crossflow Vortices.” J. Fluid Mech. Vol. 525, pp. 275-308, 2005.

19. Wassermann, P., and Kloker, M., "Mechanisms and passive control of crossflow-vortex-induced transition in a threedimensional boundary layer," J. Fluid Mech., vol. 456, pp.49-84, 2002.

20. Crouch, J.D., "Modeling Transition Physics for Laminar Flow Control,” AIAA Paper 2008-3832, 2008.

21. Balachandar, S., and Streett, C. L. \& Malik, M. R. "Secondary Instability in rotating-disk flow," J. Fluid Mech., vol. 242, pp. 323-347, 1992. 


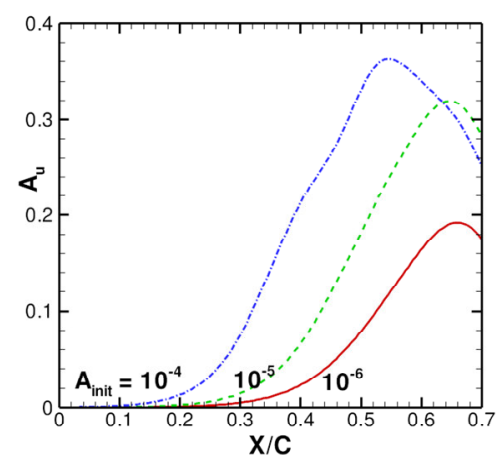

(a) $\lambda=5 \mathrm{~mm}$

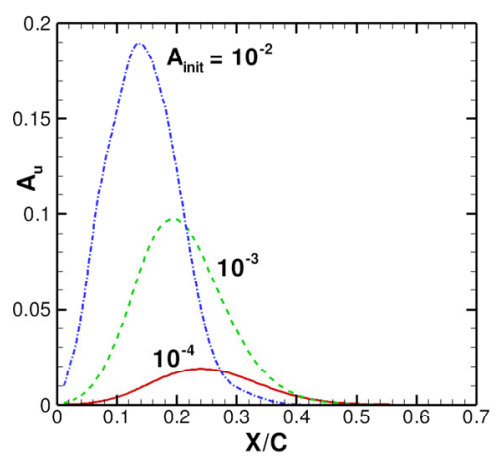

(b) $\lambda=2.25 \mathrm{~mm}$

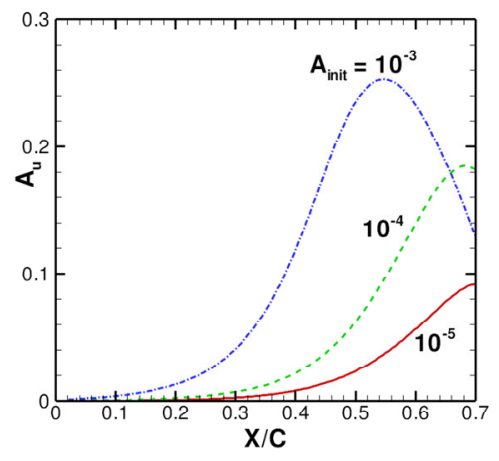

(c) $\lambda=10 \mathrm{~mm}$

Fig. 1. Nonlinear evolution of fundamental disturbance amplitude for a stationary crossflow mode at specified spanwise wavelength, with the initial disturbance amplitude as a parameter

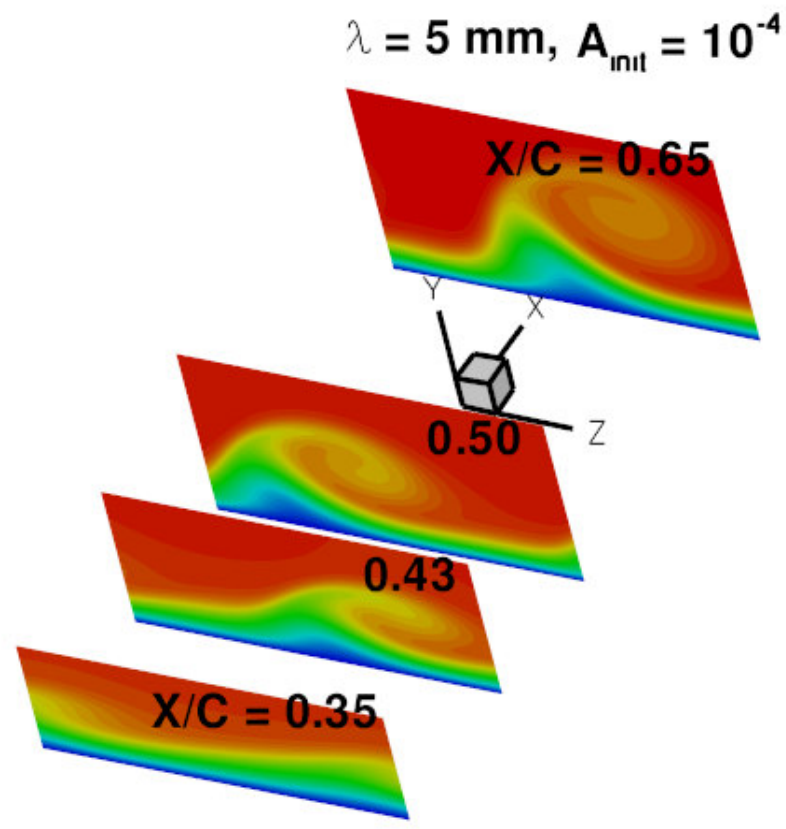

Fig. 2. Chordwise velocity contours across vortex cross section extending over one spanwise wavelength $\left(\lambda=5 \mathrm{~mm}, \mathrm{~A}_{\mathrm{i}}=1 \mathrm{e}-4\right)$ 


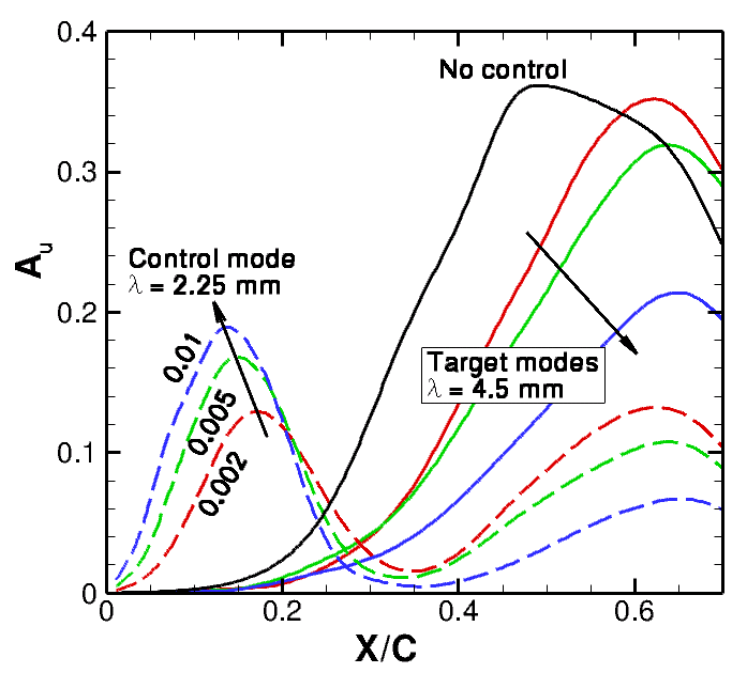

(a) Amplitudes plotted in linear scale

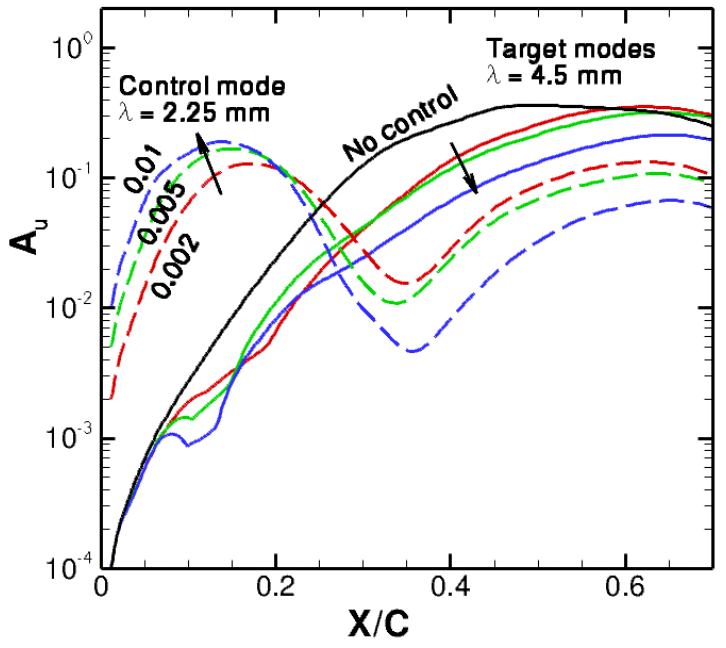

(b) Amplitudes plotted in logarithmic scale

Fig. 3. Modal amplitude evolution of linearly most unstable stationary crossflow mode in the presence of control input at $\lambda=2.25 \mathrm{~mm}$

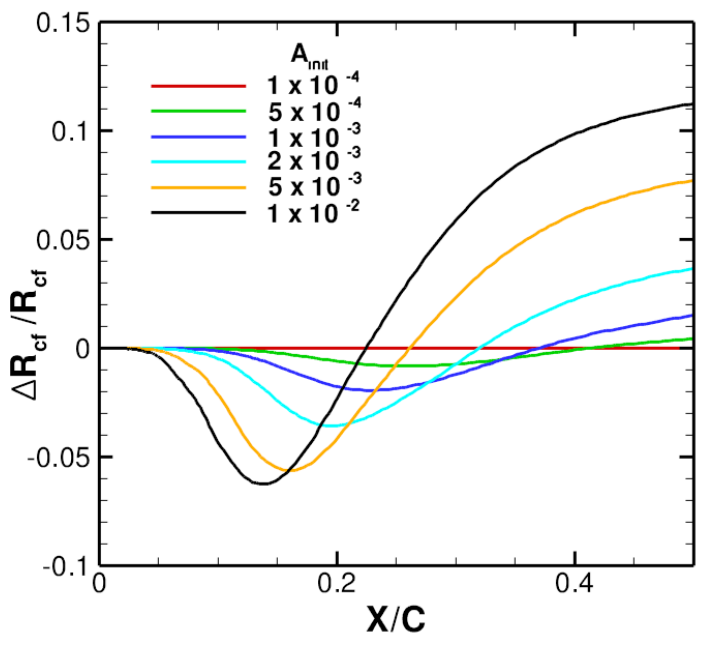

(a) Relative change in crossflow Reynolds number distribution as a result of control input at various levels

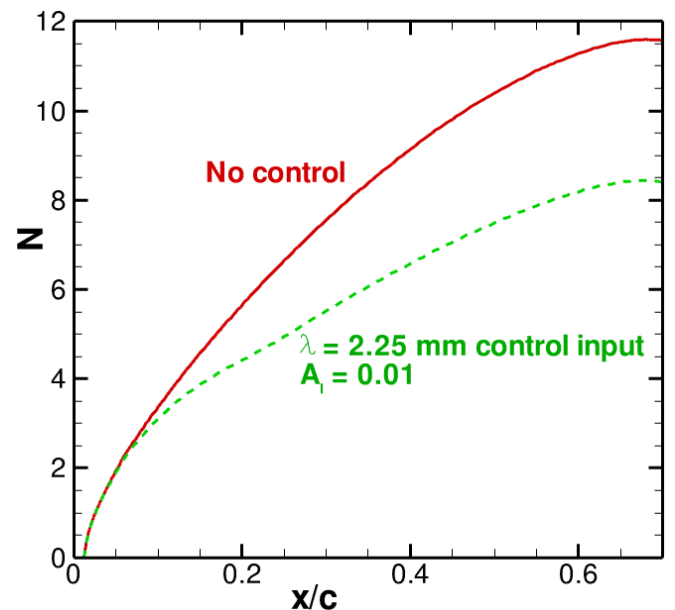

(b) Logarithmic amplification ratio ( $\mathrm{N}$-factor) of naturally dominant stationary crossflow mode $(\lambda$ $=4.5 \mathrm{~mm}$ ) with and without the mean flow correction due to control input with an initial amplitude of $A_{i}=0.01$

Fig. 4. Effect of control input $(\lambda=2.25 \mathrm{~mm})$ on instability characteristics of spanwise averaged flow 

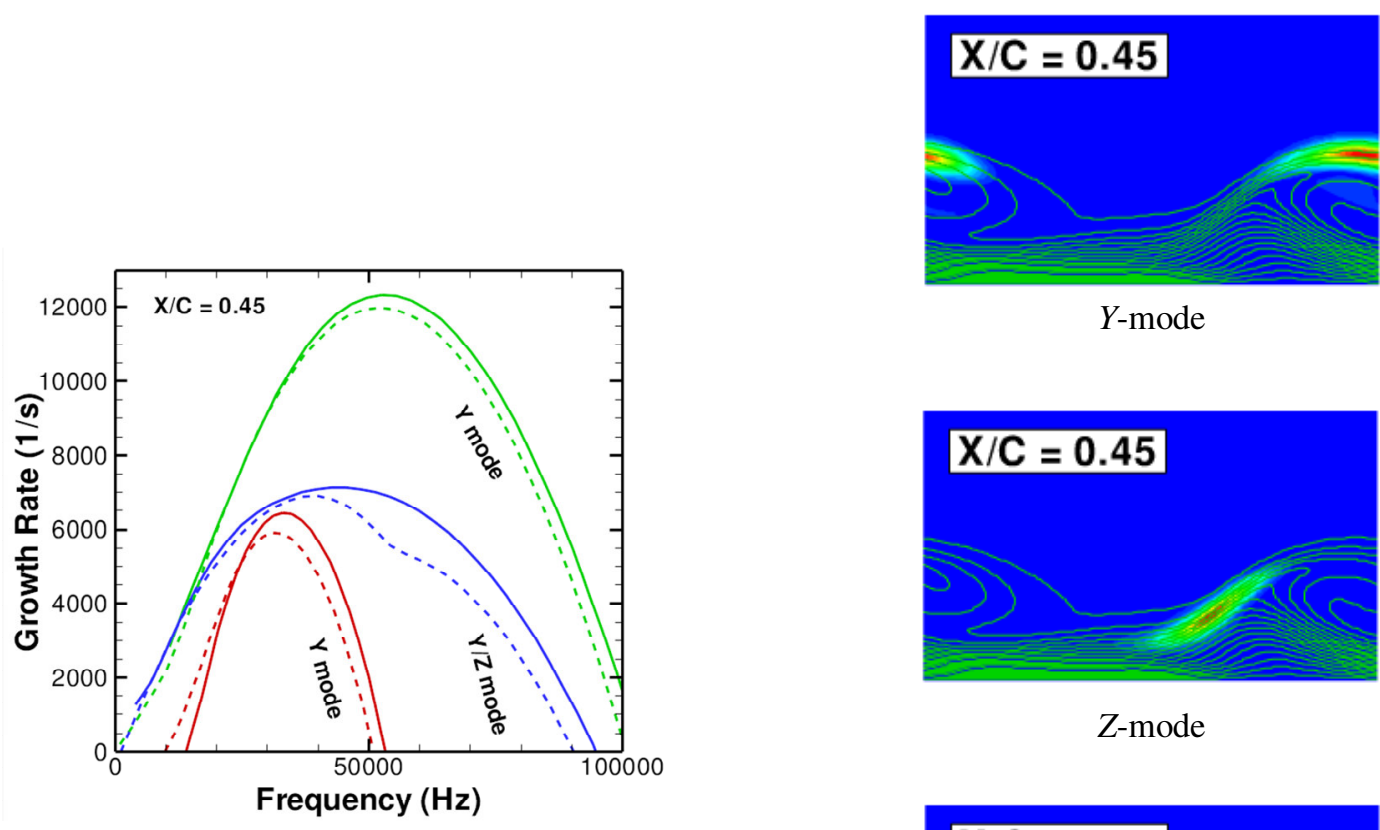

(a) Temporal growth rates with (solid lines) and without (dashed lines) $\mathrm{V}_{0}$ and $\mathrm{W}_{0}$

$Y$-mode

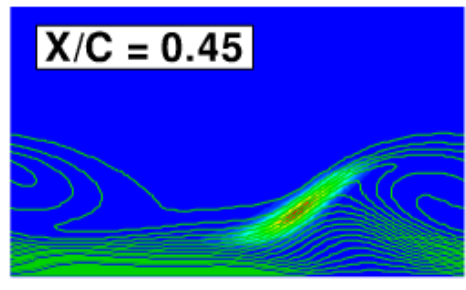

Z-mode

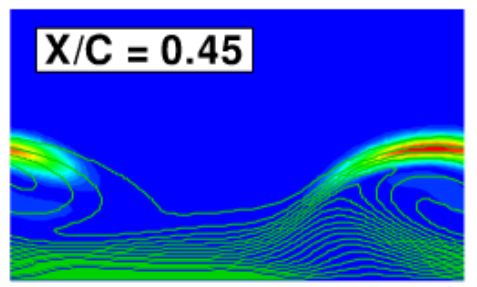

Y/Z-mode

(b) Mode shapes for three dominant families of modes

Fig. 5. High-frequency secondary instabilities at $\mathrm{x} / \mathrm{c}=0.45$ (primary crossflow mode corresponds to spanwise wavelength of $\lambda=4.5 \mathrm{~mm}$ and initial amplitude of $\mathrm{A}_{\mathrm{i}}=1 \mathrm{e}-4$ )

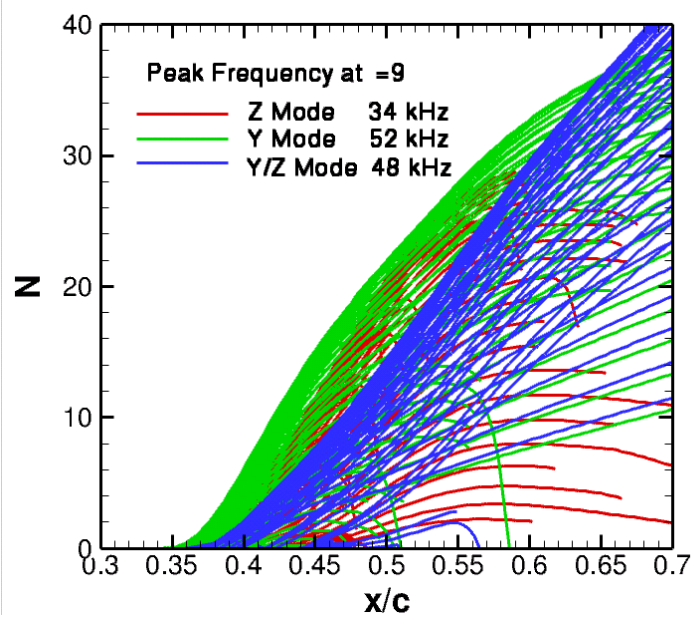

Fig. 6. N-factor curves for spatially growing disturbances at selected frequencies within each family of highfrequency secondary instabilities (indicated by different colors as described in the legend).

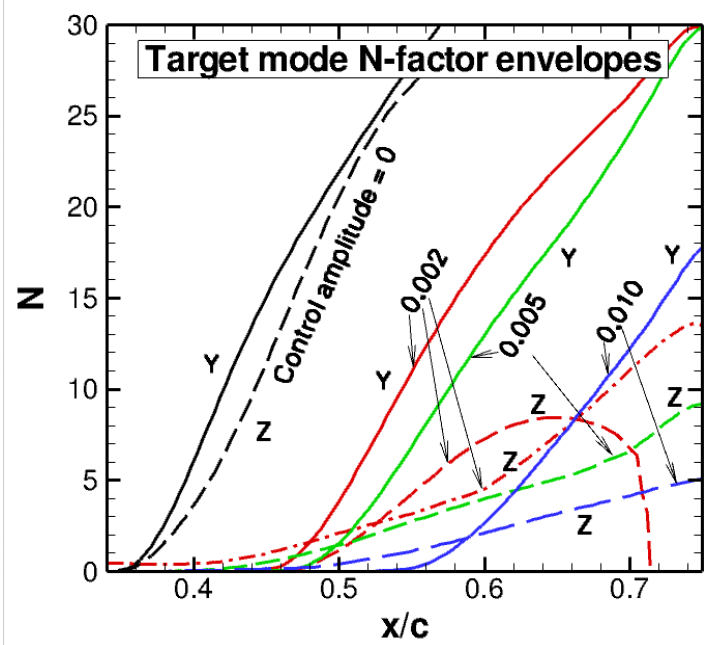

Fig. 7. Envelopes of $\mathrm{N}$-factor curves for dominant $\mathrm{Y}$ and $\mathrm{Z}$ families of secondary modes at increasing levels of control amplitude. 

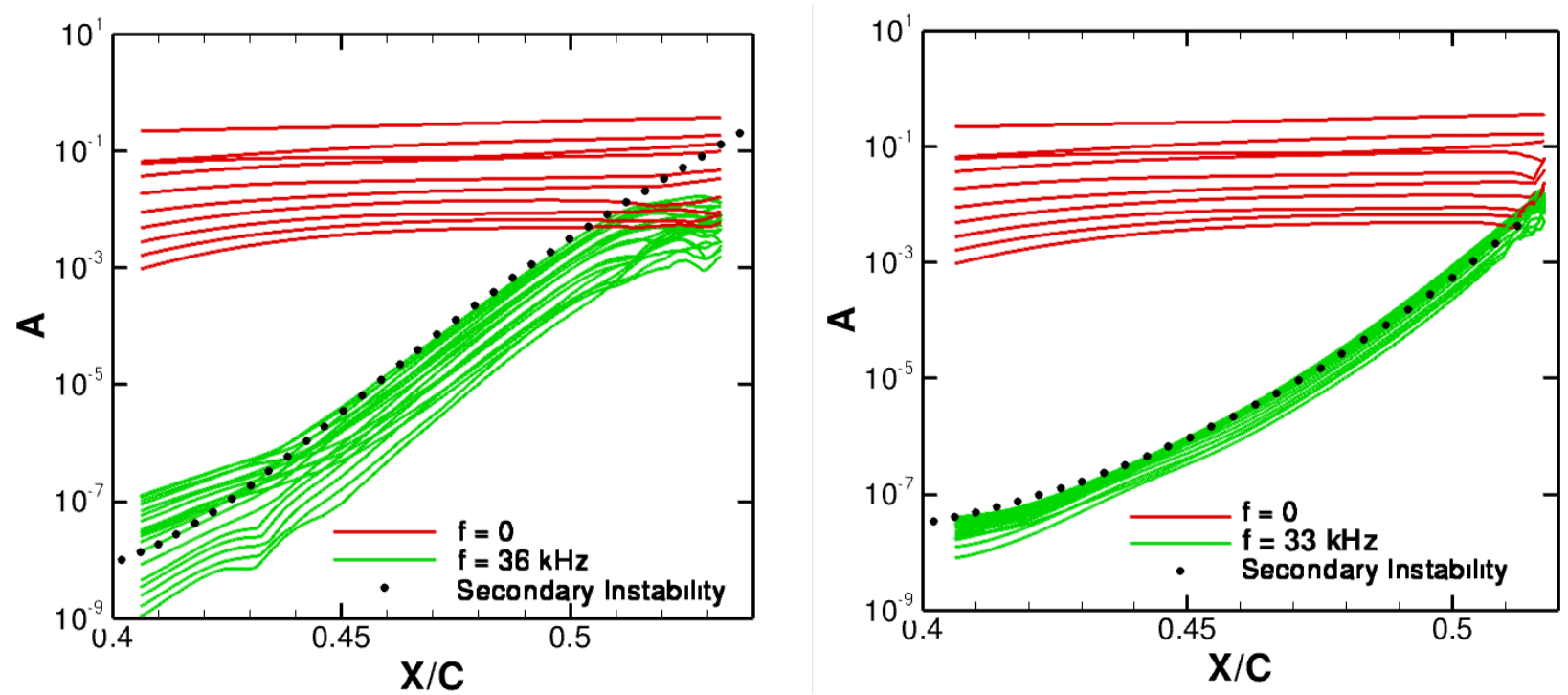

Fig. 8. Amplitude evolution of primary and secondary instability perturbations. Each line is associated with a fixed spanwise wavenumber. The red lines represent stationary modes (predominantly associated with primary crossflow instability) and the green lines represent secondary instability modes at the fundamental frequency (higher frequency harmonics are not shown). The black dots represent results from linear secondary instability theory. The y-mode secondary instability is shown on the left, and the z-mode on the right.

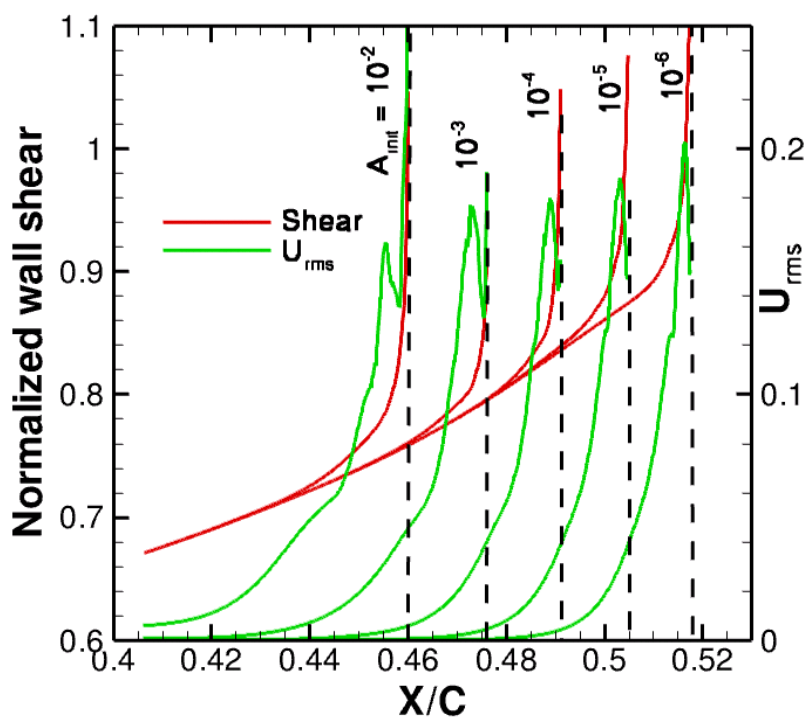

Fig. 9. Evolution of wall shear and secondary instability RMS amplitude for Z-mode secondary instability as control amplitude varies. The dashed vertical lines indicate the transition locations based on the wall-shear criterion.

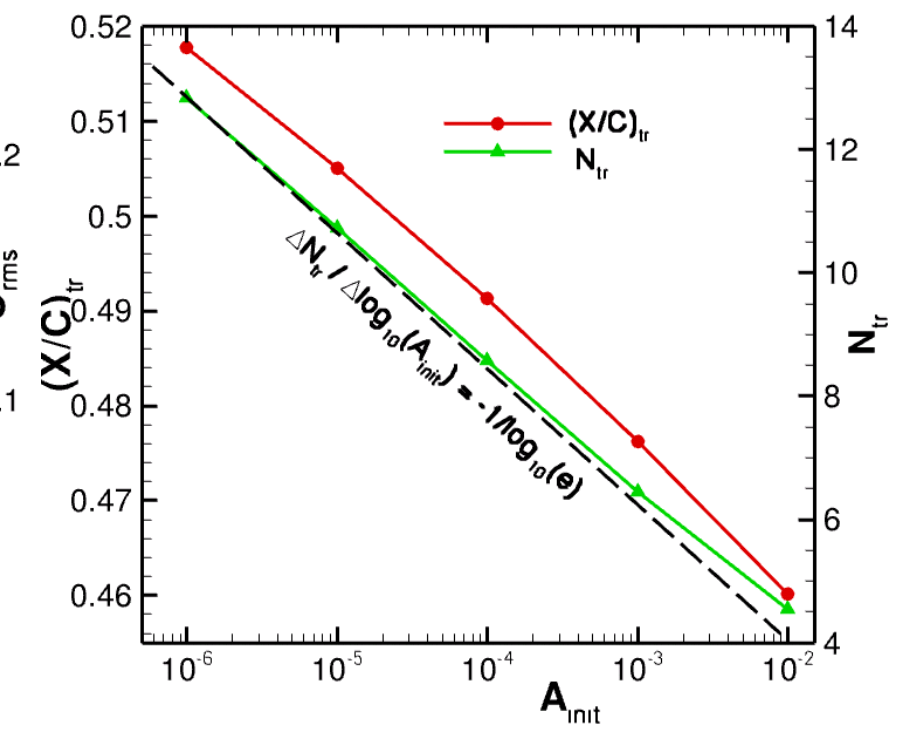

Fig. 10. Transition location and transition $\mathrm{N}$-factor as function of logarithm of secondary instability initial amplitude is almost a straight line. The latter falls approximately on a straight line with a slope given by Eq (2). (Note: the expression indicating the slope is equivalent to $\mathrm{Eq}(2)$, but is expressed in logarithm to the base 10 instead of natural logarithm to be consistent with the scaling of the horizontal axis). 

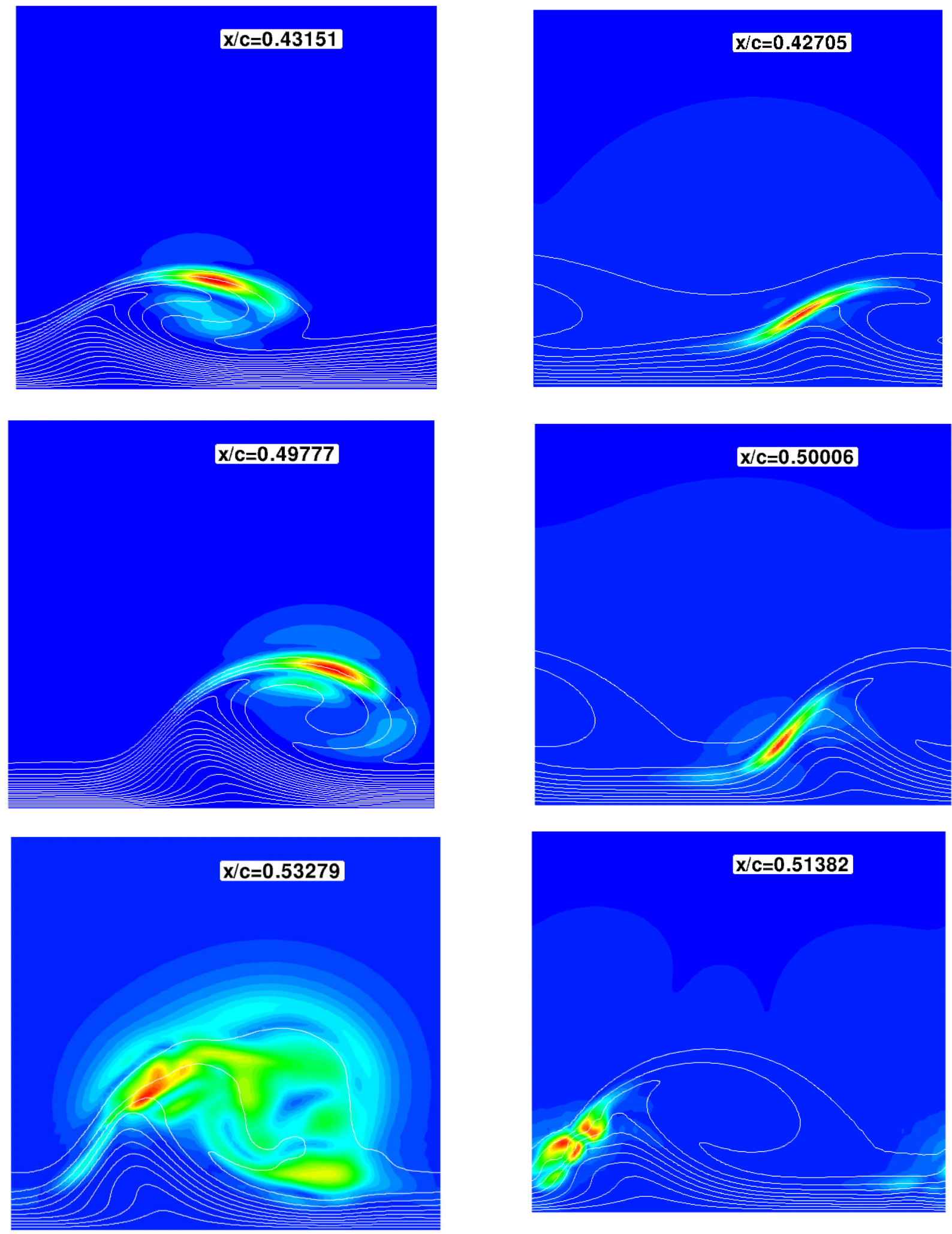

Fig. 11. Time-RMS distributions of chordwise velocity distributions at selected chordwise stations. The white lines in the background are the u-contours of stationary crossflow vortex. The y-mode is displayed on the left and the z-mode on the right. Secondary instability initial amplitude is 1e-6. 

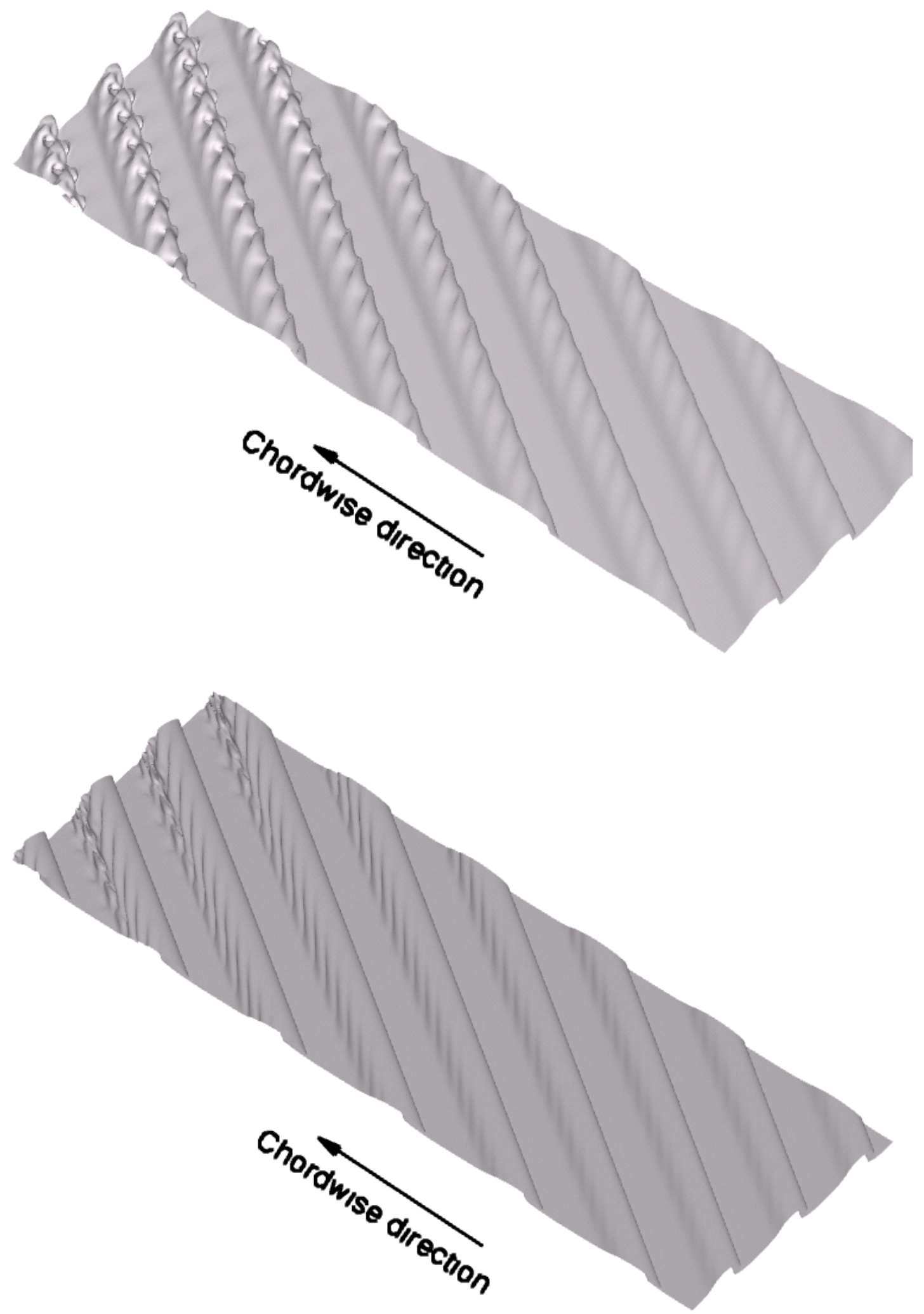

Fig. 12. Iso-surfaces of instantaneous chordwise velocity $U$. Top: $Y$-mode, $U=0.91 U_{\infty}$. Bottom: $Z$-mode, $U=0.87 U_{\infty}$ 